\title{
LAS PROFESIONES EN ESCENA: RESEÑA DE UNA TESINA SOBRE EDUCACIÓN Y TRABAJO EN CIENCIAS DE LA EDUCACIÓN
}

\author{
María Eugenia Vicente (Universidad Nacional de La Plata)* \\ mevicente@fahce.unlp.edu.ar \\ Alicia Inés Villa (Universidad Nacional de La Plata)** \\ alivilla@gmail.com
}

Recibido: 29/07/2012 Aceptado: 12/09/2012

\section{Resumen}

Se reseña una tesina que pretende aportar al conocimiento de la relación entre educación y trabajo en Ciencias de la Educación, en el marco de la reconfiguración de su campo profesional gestada en las últimas décadas. Para ello, el trabajo se separa de aquellos estudios que reconocen el sistema productivo como determinante de la formación de profesionales en educación, como de aquellos que suponen al graduado despojado de contexto y condicionantes. Desde la intersección entre la dinámica estructural del campo y las prácticas socio-profesionales de los graduados, y a través del prisma conceptual de trayectorias profesionales, que se realiza un análisis de un conjunto de historias de vida profesionales protagonizadas por graduadas mujeres en Ciencias de la Educación de la Universidad Nacional de La Plata. El análisis indica que las acciones socio-profesionales de los graduados tienen la capacidad de modificar la realidad.

\section{Palabras Clave}

Ciencias de la Educación - Educación y trabajo - Trayectorias profesionales Campo profesional - Graduados.

* Magíster en Dirección de Recursos Humanos (UCES). Profesora y Licenciada en Ciencias de la Educación (UNLP). Doctoranda en Ciencias Sociales (FLACSO Argentina). Becaria doctoral del Consejo Nacional de Investigaciones Científicas y Técnicas (CONICET).

** Doctora en Ciencias Sociales por la Facultad Latinoamericana de Ciencias Sociales (FLACSO Argentina); Magíster en Investigación Educativa por la Universidad Academia de Humanismo Cristiano (UAHC) de Chile; Profesora y Licenciada en Ciencias de la Educación por la Universidad Nacional de La Plata (UNLP). 


\begin{abstract}
The aim of the thesis reviewed is to contribute to the knowledge about the relationship between education and work in Education Sciences, as part of the reconfiguration of its professional field in last decades. The work is separated from those studies that recognize the productive system as a determinant of the education of professionals, and those who recognize the graduate out of context and conditions. From the intersection between the structural dynamics of the field and socio-professional practice of graduates, and through the concept of professional trajectories, these theses analyzes a set of professional life histories of woman graduates in Education Sciences from Universidad Nacional de La Plata. The analysis indicates that socio-professional activities of graduates have the ability to change reality.
\end{abstract}

\title{
Key Words
}

Education Sciences - Education and work - Professional trajectories - Professional field - Graduates.

\section{Introducción}

"Educación y trabajo en Ciencias de la Educación: aportes desde las trayectorias profesionales de sus graduados" es el título de la tesina de Licenciatura en Ciencias de la Educación aquí reseñada, evaluada y aprobada en 2012 en la Facultad de Humanidades y Ciencias de la Educación de la Universidad Nacional de La Plata.

En la primer parte del trabajo denominada "El estudio de la relación entre educación y trabajo en Ciencias de la Educación" la autora sienta su posición teórica y metodológica, realizando un recorrido analítico de la bibliografía y estudios existentes sobre el tema de educación y trabajo en Ciencias de la Educación. El recorrido permite conocer que las conclusiones se separan de aquellas que reconocen al sistema productivo como determinante de la formación de profesionales en educación, como de aquellas que suponen al graduado despojado de contexto y condicionantes. Desde la relación entre la dinámica estructural del campo y las prácticas socio-profesionales de los graduados que el estudio interroga por las prácticas y estrategias de los graduados a lo largo de sus trayectorias.

En la segunda parte del trabajo, denominada "Las trayectorias profesionales de los graduados en Ciencias de la Educación como objeto de estudio" se presenta el análisis de las historias de vida profesionales de los graduados en Ciencias de la Educación en relación a un conjunto de dimensiones: la propia biografía escolar y la reflexión sobre ella, las acciones (e innovaciones) pedagógicas emprendidas en los distintos ámbitos laborales, las resistencias y reacciones ante dichas acciones, el contexto o condiciones del mercado laboral, y el lugar de las mujeres en clave de la dimensión de género para dar cuenta del fenómeno de feminización de la profesión. 


\section{El campo profesional de las Ciencias de la Educación}

El problema de indagación que da origen a la tesina reconoce los cambios en el campo profesional a lo largo de las últimas décadas. Por ello, se realiza un balance entre pasado y presente de la carrera de Ciencias de la Educación a la luz de los espacios de acción socio-profesional.

En la historia de la institucionalización y profesionalización de las Ciencias Sociales en Argentina, las Ciencias de la Educación son una de las disciplinas primeras en institucionalizarse con la apertura de una facultad y carrera, junto a la publicación de una revista especializada en educación. En 1914, se funda la carrera y la Facultad de Ciencias de la Educación en la Universidad Nacional de La Plata, publicando ese mismo año el primer número de la Revista Archivos de Ciencias de la Educación (1).

No obstante la presencia histórica de los profesionales en Ciencias de la Educación en el campo mayor de las Ciencias Sociales, en la actualidad se presenta como un campo profesional en disputa y reconfiguración. Aquello que podría considerarse legítimo y reconocerse como propio del campo es altamente amenazado desde la década del 70 , cuando se desligan las Ciencias de la Educación de la docencia como opción principal de ejercicio profesional, presentando intereses socio profesionales muy diversos. Con ello, profesionales de otras disciplinas sociales (sociólogos, psicólogos, entre otros), mantienen importantes intereses en la educación y sientan luchas y acciones que mantienen la ruptura del monopolio de la intervención educativa por parte de sus especialistas, los graduados en Ciencias de la Educación.

De esta manera, el núcleo problemático del estudio refiere a que el campo profesional de las Ciencias de la Educación no sólo se encuentra en reconfiguración al redefinir sus vínculos con los espacios tradicionales de acción profesional, sino que además es un campo disputado por otras profesiones.

\section{Perspectivas y discusiones en torno de la relación entre educación y trabajo en Ciencias de la Educación}

Luego de reconocer, a modo introductorio, que el problema que convoca a la tesina refiere al tema sobre educación y trabajo en Ciencias de la Educación, una importante parte del trabajo se aboca a presentar y analizar los aportes que se vienen desarrollando al respecto, particularmente respecto de los egresados en Pedagogía de la Universidad de Barcelona (España), de la Universidad Nacional Autónoma de México y de la Universidad Veracruzana (México); y de los graduados en Ciencias de la Educación de la Universidad Nacional de Cuyo, de la Universidad Nacional de San Luis, de la Universidad Nacional de Luján, de la Universidad Nacional de Salta y de la Universidad Nacional de Jujuy (Argentina). La autora los ubica en torno de tres perspectivas: estructuralista, subjetivista y de trayectorias profesionales. 
En una primera perspectiva de tipo estructuralista, el perfil profesional demandado por el mercado de trabajo se constituye no sólo en referente de la formación a realizar, sino también se lo reconoce como el resultante de las necesidades que hay que satisfacer. Por ello, los estudios enmarcados en esta línea conllevan el propósito de indagar las habilidades y conocimientos requeridos por los empleadores, para luego adaptar el plan de estudios/formación de las carreras a las necesidades observadas en el mercado de trabajo. En este sentido, las preguntas que ayudan a responder son ¿Cuánto hay de correspondencia entre el perfil del graduado en Ciencias de la Educación y la demanda laboral? En función de ello, ¿qué y cómo mejorar la formación del graduado en Ciencias de la Educación?

La autora reconoce que si bien esta perspectiva brinda un panorama acerca de la estructura del mercado laboral y sus demandas a la educación, una segunda mirada sobre la relación entre educación y trabajo sostendrá que, retomando los aportes de María de Ibarrola (2004), François Dubet (1994), Pierre Bourdieu y Loïc Wacquant (2005), y Alicia Gutierrez (2001), la función social de la educación no se reduce a ser la proveedora puntual de los recursos humanos que supuestamente requieren los diferentes mercados de trabajo. Asimismo, en relación con el lugar del graduado, los agentes sociales no son partículas mecánicamente movilizadas por fuerzas externas, los actores también tienen capacidad de opción, de elección y de reflexión sobre lo que hacen. Discusión que habilita a la pregunta ¿cómo construyen el campo profesional de las Ciencias de la Educación los propios graduados?

La pregunta anteriormente planteada es rescatada en una segunda perspectiva, anclada en los conceptos de representaciones, expectativas, anticipaciones, resignificaciones. Las decisiones que los sujetos tomen de cara a su futuro profesional, ya sean graduados o estudiantes avanzados, están orientadas según las representaciones que se den de sí y las expectativas respecto de su profesión. La tesista sostiene que los estudios analizados en esta perspectiva ayudan a responder preguntas tales como ¿cuáles son las anticipaciones respecto del funcionamiento del mercado del empleo, de los profesionales del área? ¿Cuáles son los rasgos que han ido constituyendo la identidad de los pedagogos? ¿Cuáles son los significados atribuidos al campo profesional de Ciencias de la Educación durante el proceso de formación? ¿Cómo se relacionan los perfiles socio-demográficos de los estudiantes con las expectativas laborales? No obstante, se advierte, resta avanzar en el conocimiento sobre la relación entre sujeto y estructura. Esto es, conocer de qué manera las representaciones, intereses, expectativas configuran estrategias socio-profesionales concretas que permitan a los graduados, a lo largo de sus trayectorias, (re) construir el campo profesional mismo caracterizado por la difusión de su objeto de intervención y la presencia de los intereses de otros profesionales. Entonces, y aunando de alguna manera los aportes de las dos perspectivas anteriores, se hace presente la pregunta: ¿Cómo se relaciona la 
estructura particular del campo profesional de las Ciencias de la Educación, y las acciones de sus graduados?

La pregunta inaugura una tercera perspectiva que, desde el enfoque de trayectorias profesionales, es abonada por la línea de autores franceses como Pierre Bourdieu (1997, 2004), Claude Dubar (2002), Loïc Wacquant (2005) y Christine Delory - Momberger (2009). Las trayectorias profesionales se constituyen a partir de las prácticas y estrategias de los graduados en torno de lógicas de acción que corresponden a elementos del sistema y se han impuesto a los actores. Pero al mismo tiempo, la dialéctica continua de esperanzas subjetivas y oportunidades objetivas que opera a lo largo de todo el mundo social puede arrojar una variedad de resultados que van desde la perfecta adecuación mutua (cuando la gente desea aquello a lo que está objetivamente destinada) hasta la dislocación radical.

Las investigaciones reseñadas en esta perspectiva demuestran que las trayectorias profesionales se pueden abordar a partir de una estrategia en particular como la realización de un posgrado, o desde un ámbito de inserción profesional determinado con incidencia de los saberes construidos en espacios laborales previos.

La tesista señala que este análisis supone identificar el "interés" propio como principio a partir del cual el agente social estructura su acción, que se convierte así en un medio (estrategia) a través del cual busca mantener o mejorar su posición. Interés que definen en relación a una posición social, las "condiciones objetivas" constituidas por dos dimensiones: una externa, (el campo) y lo incorporado por el individuo como resultado del proceso de socialización (habitus). No obstante, y retomando los interrogantes previos, aún queda por develar de qué manera las estrategias socio-profesionales impactan en la configuración y/o reconfiguración del propio campo profesional, entendiendo que las acciones de los graduados, en tanto actores sociales, tienen la capacidad de modificar la realidad. De esta manera, las trayectorias profesionales en Ciencias de la Educación se consolidan como objeto de estudio.

\section{Las trayectorias profesionales en Ciencias de la Educación como objeto de estudio}

Como planteábamos en el apartado anterior, la decisión de avanzar en el estudio de las trayectorias profesionales en Ciencias de la Educación es producto de reconocer que las prácticas, acciones y estrategias sociales que sus graduados desarrollan a lo largo de su historia profesional y en el marco de determinados contextos, están condicionadas pero también tienen capacidad de opción, de elección y de reflexión sobre lo que hacen. De esta manera, ¿Cuáles son las estrategias socio-profesionales de los graduados en Ciencias de la Educación en el marco de un campo profesional disputado y en reconfiguración? es la pregunta que guía el diseño metodológico, orientado a 
reconstruir y analizar las prácticas, experiencias y estrategias que constituyen las trayectorias profesionales a partir de las voces de sus propios protagonistas, los graduados. Se analizan cuatro historias de vida profesionales de graduados en Ciencias de la Educación de la Universidad Nacional de La Plata (UNLP) en las décadas de 1980, 1990 y 2000, a partir de reconocer puntos de inflexión históricos y personales: la identificación de relaciones, procesos, vínculos causales, contradicciones, cambios en significados. El análisis de las entrevistas se constituye en cinco dimensiones socio-profesionales.

En primer lugar, las trayectorias de los graduados entrevistados se presentan como "biografías en referencia" que tratan sobre aquellos referentes en los que el graduado se apoya o reconoce a lo largo de su vida profesional, y que van desde un importante apego a lo establecido, desde las normas, hacia la búsqueda de nuevas maneras de hacer y pensar la práctica. En un primer y segundo momento de la historia profesional del graduado, el referente de su práctica se va desligando de la norma, del deber ser, y se torna hacia las prácticas y sujetos concretos. Giro clave para la construcción de nuevos espacios de acción pedagógica o la consolidación de otros que están emergiendo, o bien para el desarrollo de innovadoras propuestas pedagógicas. Y ello se relaciona con un tercer momento, que implica abandonar la idea de sujeto como ejecutor de normas, para reconocerse constructor y diseñador de su propio espacio de acción. Se trata de espacios emergentes como la acción pedagógica en espacios de ocio y recreación, en propuestas de museos y exposiciones, en colonias de vacaciones, entre otros.

En segundo lugar, se trata del lugar de las biografías escolares en las trayectorias de los graduados. Son rescatados los aportes de Cristina Davini (1995), quien reconoce que las biografías se presentan como el resultado de complejas internalizaciones durante la vida escolar que van generando un "fondo de saber" que regula las prácticas. Desde esta base conceptual, la autora discute de alguna manera con los aportes al respecto de Alejandra Birgin (1997), Gabriela Diker y Flavia Terigi (2003), quienes sostienen que en las instituciones de inserción socio laboral dichas historias son reforzadas y reproducidas, existiendo un continum entre la biografía escolar del futuro enseñante, su formación como docente y la escuela en la que se inserta como profesional. Las instituciones aparecen como "cómplices" en la reproducción de los esquemas tradicionales, logrando un círculo reproductivo. No obstante, el análisis de los entrevistados intenta demostrar que las biografías escolares pueden ser puestas en discusión apuntando a redefinir el sentido de las prácticas profesionales.

En tercer lugar, la propia concepción de profesión repensada a partir de la idea de individuo y cargo. Se reconoce al individuo como actor social, creador de su propio recorrido profesional y con ello el cargo o rol en tanto espacio de posibilidades, en contraposición con la existencia de roles y cargos definidos 
a priori. Las acciones socio-profesionales narradas por las entrevistadas dan cuenta que es posible trabajar en orden a generar las necesidades sociales de los profesionales en Ciencias de la Educación, y no sólo esperar las demandas dependiendo únicamente de los empleadores o clientes que buscan controlar los términos, las condiciones y el contenido de los trabajos que desean les realicen. Así, es a partir de los aportes de Eliot Freidson (2001), que la autora concibe el enfoque sobre la profesión desde la idea de profesionalización de las Ciencias de la Educación para intentar dar cuenta de la idea de devenir constante de la profesión en toda acción pedagógica de cada graduado.

En cuarto lugar, respecto de las condiciones y reacciones del entorno social, particularmente se trata del análisis sobre las condiciones de trabajo y sobre las reacciones que despiertan los cambios emprendidos por graduados en determinados ámbitos laborales. Anclada en la idea de un "estado de relación de fuerzas" entre distintos agentes que actúan en un mismo espacio, las nuevas acciones pedagógicas desarrolladas por las entrevistadas en distintos espacios laborales presentan resistencias y reacciones por parte de uno o más sujetos: la intención de disolver un grupo de trabajo, denostar la imagen de un profesional en la institución donde labora, son algunas manifestaciones de dichas reacciones. Al tiempo que la gestión institucional actúa como facilitador u obstaculizador de los cambios emprendidos por los graduados en las instituciones.

Respecto de las condiciones de seguridad laboral, si bien el trabajo no considera que exista una relación directamente proporcional entre nuevos espacios de acción pedagógica y la ausencia de regulación que impacte negativamente en la seguridad laboral, sí es posible reconocer que la construcción de nuevos espacios también debe atender a la cuestión de las condiciones de trabajo. El análisis avanza en ello debido a que reconoce que los graduados se emplean para tareas de corta duración, de algunos meses, haciendo de estos profesionales "interinos permanentes", situación que acoge la instalación de la precariedad.

En quinto lugar, respecto de la presencia de la mujer en el campo profesional de las Ciencias de la Educación, la dimensión de género posibilita superar el tradicional análisis acerca de que el lugar de la mujer en el campo de la educación fue un permiso concedido por la sociedad y regulado por el Estado donde su vida familiar, su moral y conducta en el ámbito privado se le permitía extenderse al ámbito público, "maternando" a los niños en las aulas. Más bien, el estudio reconoce que se trata de espacios (re) pensados, (re) definidos y (re) construidos por las mujeres en un marco de acción pedagógica y social donde los espacios no necesariamente existen a priori. Particularmente desde el ángulo analítico de la categoría de género, se reconoce en la mujer el poder de redefinición de la realidad y la consecuente inauguración de espacios inexplorados, situación que revierte el destino inexorable del sexo femenino en la 
educación, orientada más bien hacia una conquista constante de las mujeres en la reinvención del campo profesional de las Ciencias de la Educación, guiadas ya no por la disposición, sino por la elección.

A modo de cierre, reconocemos que se trata de una tesina que visibiliza historias, imágenes, huellas, momentos y oportunidades como resultados de acciones y decisiones que marcan debates y combates con otros profesionales y otras profesiones, con gestiones institucionales y regulaciones laborales, y con la propia historia de la profesión. En este sentido, el estudio posibilita reconocer al graduado en Ciencias de la Educación como diseñador de la profesión, a partir de trazar y volver a trazar sentidos, espacios, posibilidades y respuestas. Construyendo él mismo la demanda social de las Ciencias de la Educación.

\section{Notas Bibliográficas}

(1) Se recuerda que en 1658, se estructuró la Educación como ciencia autónoma a partir del establecimiento de los principios fundamentales de la Pedagogía, por parte de Juan Amós Comenio. Posteriormente, en el siglo XVIII la economía se constituye como campo de intervención; en 1957, tuvo lugar la institucionalización de la sociología como disciplina universitaria, y hacia la mitad de 1960, nace la psicología en tanto campo disciplinar.

\section{Referencias Bibliográficas retomadas de la tesina reseñada}

- Birgin, A. (1997) Las regulaciones del trabajo de enseñar. Vocación, estado y mercado en la configuración de la docencia. Buenos Aires: FLACSO.

- $\quad$ Bourdieu, P. (1997) Razones Prácticas. Barcelona: Editorial Anagrama.

- $\quad$ Bourdieu, P. (2004) El baile de los solteros. Madrid: Editorial Anagrama.

- Bourdieu, P. y Waqcuant, L. (2005) Una invitación a la sociología reflexiva. Buenos Aires: Siglo XXI Editores.

- Davini, M. C. (1995) La formación docente en cuestión: política y pedagogía. Buenos Aires: Editorial Paidós.

- De Ibarrola, M. (2004) ¿Qué formación para el trabajo en la educación secundaria y terciaria? en Jacinto, C. ¿Educar para qué trabajo? Discutiendo rumbos en América Latina, (pp. 335-352) Buenos Aires: La Crujía.

- Delory - Momberger, Ch. (2009) Biografía y educación. Figuras del individuo - proyecto. Buenos Aires: Editorial de la Facultad de Filosofía y Letras de la Universidad de Buenos Aires; Consejo Latinoamericano de Ciencias Sociales.

- Diker, G. y Terigi, F. (2003) La formación de maestros y profesores: hoja de ruta. Buenos Aires: Paidós.

- Dubar, C. (2002) L'articulation des temporalités dans la construction des identités personnelles: questions de recherche et problèmes d'interprétation, Temporalistes, 1(44), 2-6.

- Dubet, F. (1994) "Dimensions et figures de l'expérience étudiante dans l'université de masse" Revue Française de Sociologie, 35(4), 511-532.

- Freidson, E. (2001) Las teorías de las profesiones. Estado del arte. Perfiles Educativos, 23(093), 28-43.

- Gutierrez, A. B. (2001) Una mirada sociológica de la problematización de la cuestión: condiciones sociales, familia y vivienda. Buenos Aires. 